\title{
Changes in Low Energy Neutron Counts Rate near Ground Level Associated to Steady Rain
}

\author{
Inácio Malmonge Martin, Marcelo Pego Gomes, Matheus Carlos da Silva and Bogos Nubar Sismanoglu \\ Department of Physics, Aeronautical Institute of Technology, São José dos Campos 12228-900, Brazil
}

\begin{abstract}
Measurements of neutron count rate $(0.025 \mathrm{eV}$ to $10 \mathrm{MeV})$ at ground level were performed with a single free (He-3) tube detectorthat is located near ground level in Aeronautical Institute of Technology (ITA) São José dos Campos, SP, Brazil. The data were collected uninterruptedly since February 28, 2016 to March 11, 2016. Based on the analysis of data obtained in this period, was possible to observe the occurrence of events, backscattering for example, with significant increases in the neutron count rate during about 3 days of steady rain for all nearby regions. These events seem to be correlated with changes in local weather conditions such as cloud coverage or rain-precipitation of weak intensity. It was reported that the non-observation of a single event seems to be an indicative of the production of a burst of neutrons by a lightning discharge near the detector. Some explanations about the increasing of low energy neutrons are suggested in this article.
\end{abstract}

Key words:Neutron counts, steady rain, backscattered neutron.

\section{Introduction}

Neutron radiation is a potentially the most dangerous health hazard due to its high penetration power and ability to produce radionuclides in most substances, including the body tissues. Studies of the neutron component of the ambient radiation were primarily stimulated by concern about the exposure of aircrews to atmospheric cosmic radiation [1]. At commercial aviation altitudes the neutron component of the secondary cosmic radiation constitutes about half of the dose equivalent. Ambient neutrons at the ground level also pose a threat for semiconductor devices causing soft-errors of Static Random Access Memory (SRAM) and Dynamic Random Access Memory (DRAM) units [2]. Due to a constant and fast rise of semiconductor devices in use today, it becomes a serious problem worldwide.

It was assumed initially that the neutron flux observed at the ground level is constituted of the energetic particles coming directly from the overlying air or those backscattered (albedo) from the terrestrial

Corresponding author: Inácio Malmonge Martin, Ph.D., main research fields: magnetic field, radiation and solar plasma. surface. The neutrons of the crust origin were considered priori markedly less than those from the cosmic rays. A discovery of a link between atmospheric radon concentration and seismologic activity [3] increased interest for studies of the crust origin radiation. Subsequent studies of a near-ground neutron flux demonstrate that a flux of the crust neutrons is comparable to or even exceeding that originating from cosmic rays.

Observations of the natural neutrons are a promising basis for development of technics of environmental monitoring including seismology activity [4], hydrology [5] and possibly climate change or space weather. Linked to this is the need for more accurate determination of the origin from the near ground neutrons variations in time and energy.

\section{Material and Methods}

For neutron measurements reported in this paper it was used a neutron detector through a tube gas Ludlum based on a He-3 tube (LND, USA; model 25311) with an sensitive area of approximately $250 \mathrm{~cm}^{2}$ and covered with paraffin (radii of $9 \mathrm{~cm}$ ). That was used to detect and record thermal, epithermal and fast neutron 
at ground level in the energy range of $0.025 \mathrm{eV}$ to 10 $\mathrm{MeV}$, during January/February/March of 2014 in São

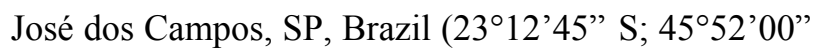
$\mathrm{W})$. The detector and other hardware are housed in a container and placed above ground level in Institute of Aeronautical and Space-IAE (Fig. 1).

The analog signals are converted into digital signals by an embedded circuit (PMI-30) which also feeds the He-3 tube that permits to vary the voltage from 1,200 VDC to 2,000 VDC, where technical details are on the US company's website (Aware Electronics Inc.-USA). Both tests and trials were carried out with the tube and electronic system associated with data acquisition using a source of 241 (Am-Be) neutron laboratory. The signs of counts have been accumulated for a time of 1 minute and save the time from the beginning and the count verified that minute, in a txt file. To study the neutron background radiation in this energy range in the equatorial and tropical regions with sensitive area of tube $\sim 250 \mathrm{~cm}^{2}$ sampling time of 1 minute that give the best performance. Equipment with "no break power supply" installed in the shelter guarantee the stability of electricity over a period of 4 hours continuously to provide energy in the case of offline provider network. The temperature inside the shelter was maintained throughout the period of measurement in the amount of $(20 \pm 1){ }^{\circ} \mathrm{C}$. From January 2012, this experimental set was always on monitoring the intensity of neutrons present in applied meteorology center (ACA) of Institute of Aeronautics and Space (IAE), in São José dos Campos, SP, Brazil [6]. The method used to make the best visualization of the measures was proposed every two days to collect the data without deleting the recording file which was attached to a Dell 630 laptop. Through Excel and Origin 8.5 software the graphics was made of the period measured showing intensity of neutrons per minute versus time. So with this chart, normal background noise radiation could be verified or if there had been in this time a different variation indicating presence of another generating source as lightning, rain and thick clouds crossing the region. To

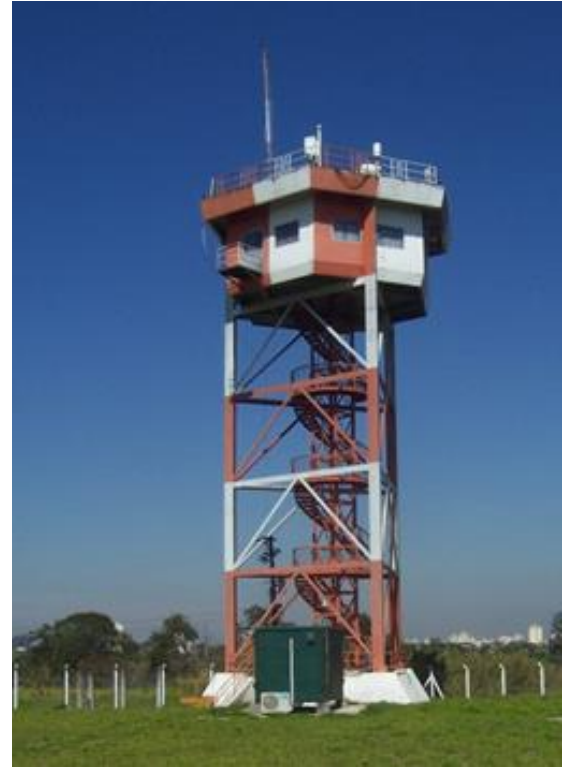

Fig. 1 Overview of container near IAE tower where is placed the neutron detection system with associated electronics.

determine periodicities in long series of intensity measurements, Fast Fourier Transform (FFT) analysis was made (power spectra) thus observing periods related to various environmental phenomena in the region [7-10].

\section{Results and Discussion}

In Fig. 2, plots the intensity of rain $(\mathrm{mm} / \mathrm{min})$ and neutron radiation (counts $/ \mathrm{min})$ on energy range $(0.025$ $\mathrm{eV}$ to $10 \mathrm{MeV}$ ) for the period of time between 00:01 h of day $02 / 28$ to $17: 47 \mathrm{~h}$ of $03 / 11$. It is noted that the observations from the second day to the ninth day of rain (Fig. 2) was measured with higher and lower intensities. Checking the corresponding measures of gamma radiation and location of the radiation intensity corresponds to the variation of rainfall intensity. This shows that the amount of water in the soil increases during all the period of rain, and correspondingly increases the hydrogen atoms on site. Hydrogen causes more neutrons thus escaping from the surface to the air by increasing the flow in the vicinity of the detector. This phenomenon (neutron backscatter) happens when the soil is wet for several days with the air close and damp. 
Soil moisture content on a horizontal scale of hectometer and at depths of decimeters can be inferred from measurements of low-energy cosmic-ray neutrons that are generated within soil, moderated mainly by hydrogen atoms, and diffused back to the atmosphere. These neutrons are sensitive to water content changes, but largely insensitive to variations in soil chemistry, and their intensity above the surface is inversely correlated with hydrogen content of the soil [11].

The FFT (Fig. 3), from the all data series of neutrons measurements period shows clearly cycles of 1 day. Certainly these cycles are caused by the presence of

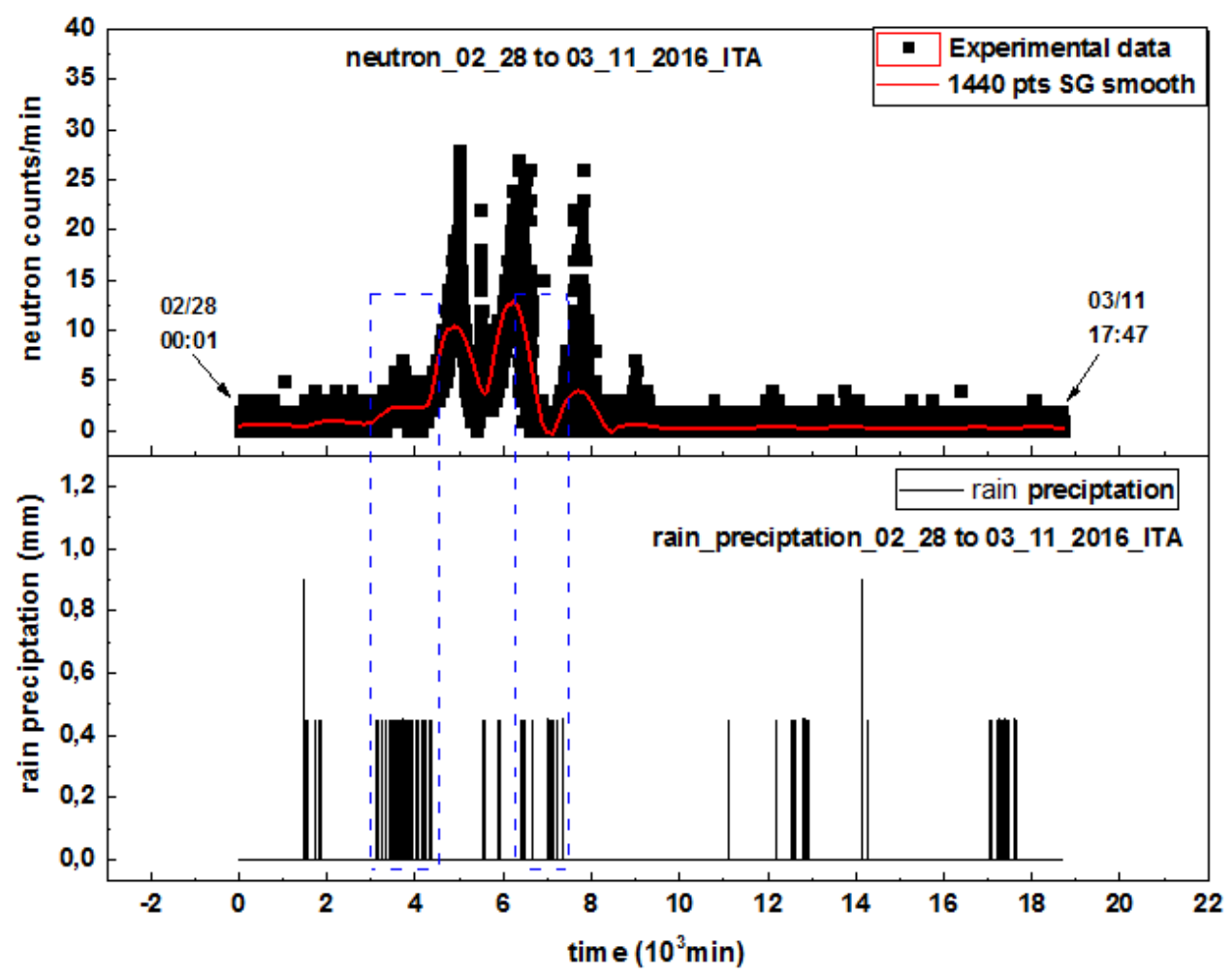

Fig. 2 Time (UT) during the period neutron (counts/min.) versus rain intensity distribution ( $\mathrm{mm} / \mathrm{min})$.

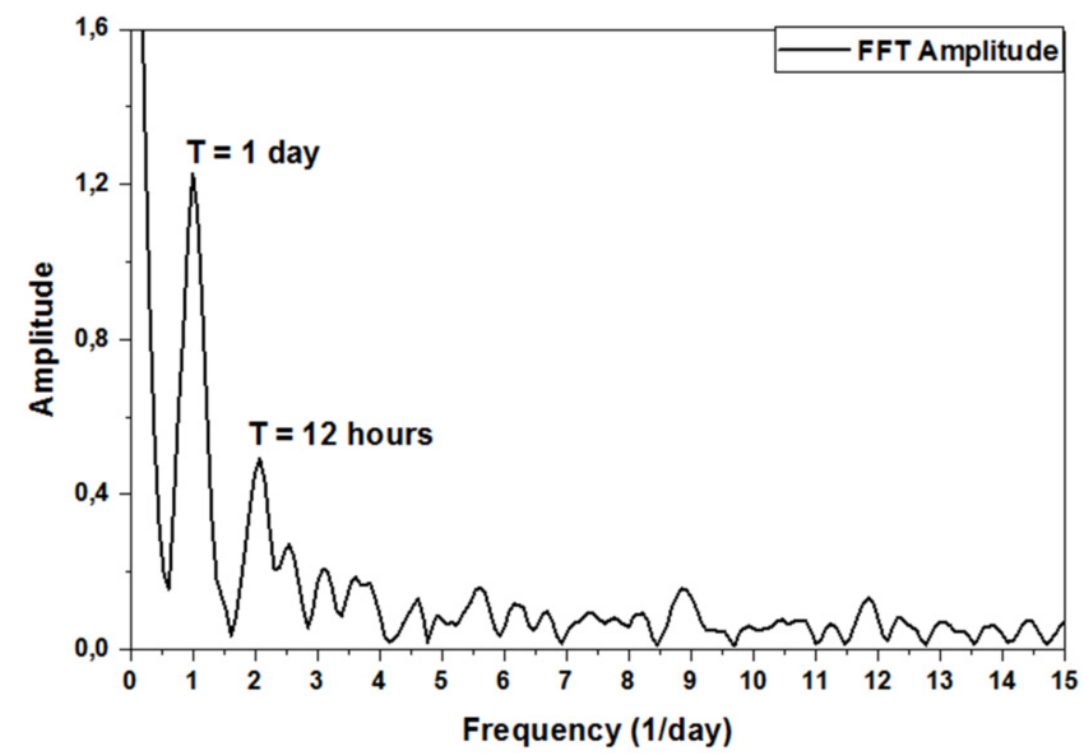

Fig. 3 Power Spectra of neutrons counter rates per day (FFT). 
radon gas due to exhalation provoked by variation of the solar insolation at the site of measurements. During the period of steady rain the intense variation of neutrons is due to hydrogen contained in water surface and moving into the air near the surface atmosphere (backscatters neutrons).

\section{Conclusions}

A simple neutron proportional counter using a (He-3) tube placed within $9 \mathrm{~cm}$ paraffin radius and associated electronica could be measured in wet soil backscatter neutrons for 9 days of steady rains in the region of São Jose dos Campos, SP and Brazil. The correlation between increases of fluxes of neutrons measured (backscatter) and period of steady rain correspond to the increases of hydrogen atom from wet soil. These phenomena can be seen once keeping the detector 1 to 2 meters above surface level.

\section{Acknowledgements}

Inácio M. Martin et al. thank CAPES-ITA and National Council for Scientific and Technological Development (CNPq) for the financial and fellowship's support.

\section{References}

[1] Nakamura, T., Uwamino, Y., Ohkubo, T., and Hara, A. 1987. "Altitude Variation of Cosmic-ray Neutrons." Health Physics 53 (5): 509-517.

[2] Martinie, S., Uznanski, S., Autran, J. L., Roche, P., Gasiot, G., Munteanu, D., et al., 2010. “Alpha-emitter Induced Soft-errorsin CMOS $130 \mathrm{~nm}$ SRAM: Real-time Underground Experiment and Monte-Carlo Simulation." Presented at the 2010 IEEE International Conference on
Integrated Circuit Design and Technology, Grenoble, France.

[3] Ulomov, V. I., and Mavashev, B. Z. 1967. "O Predvesnike Sil'nogo Tecktonicheskog Zemietryarsenia (A Precursor of a Strong Tectonic Earthquake)." Doklady Earth Sciences Sections 176: 9-11.

[4] Gregorič, A., Vaupotič, J., and Gabrovse, F. 2013. "Reasons for Large Fluctuation of Radon and $\mathrm{CO}_{2}$ Levels in a Dead-end Passage of a Karst Cave (Postojna Cave, Slovenia)." Natural Hazards and Earth System Sciences 13: 287-297.

[5] Desilets, D., Zreda, M., and Prabu, T. 2006. "Extended Scaling Factors for in Situ Cosmogenic Nuclides: New Measurements at Low Latitude." Earth and Planetary Science Letters 246 (3): 265-276.

[6] Martin, I. M., Gomes, M. P., Ferro, M. A. S., Pinto, M. L. A., and Antônio, F. C. 2013. "Measurements of X and Gamma Radiation at Ground Level and Their Correlation with Atmospheric Electric Discharges and Rainfall in São José dos Campos, SP, Brazil.” World Environment 3 (4): 138-141.

[7] Nakamura, T. 2014. "Cosmic-Ray Neutrons Spectrometry and Dosimetry." Journal of Nuclear Science and Technology 45 (5): 1-7.

[8] Chyi, L. L., Quick, T. J., Yang, T. F., and Chen, C. H. 2005. "Soil Gas Radon Spectra and Earthquakes." Tao 16 (4): 763-774.

[9] Yajima, K.,Yasuda, H., Takada, M., Sato, T., Goka, T., Matsumoto, H., et al., 2010. "Measurements of Cosmic-Ray Neutron Energy Spectra from Thermal to $15 \mathrm{MeV}$ with Bonner Ball Neutron Detector in Aircraft." Journal of Nuclear Science and Technology 47 (1): 31-39.

[10] Martin, I. M., and Alves, M. A. 2010. "Observations of a Possible Neutron Burst Associate with a Lightning Discharge in Brazil." Journal Geophysical Research 115 (A00E11): 1-4.

[11] Zreda, M., Desilets, D., Ferré, T. P. A., and Scott, R. L. 2008. "Measuring Soil Moisture Content Non-Invasively at Intermediate Spatial Scale Using Cosmic-ray Neutrons." Geophysics Research Letters 35 (L21402): 1-5. 